\title{
Absorption of Laser Light in Overdense Plasmas by Sheath Inverse Bremsstrahlung
}

\author{
T.-Y. Brian Yang, W. L. Kruer, R. M. More
}

This paper was prepared for submittal to the 36th Annual Meeting of the American Physical Society

Division of Plasma Physics

Minneapolis, $\mathrm{MN}$

November 7-11, 1994

November 1994

- This is a preprint of a paper intended for publication in a joumal or proceedings. Since changes may be made before publication, this preprint is made available with the understanding that it will not be cited or reproduced without the permission of the author.

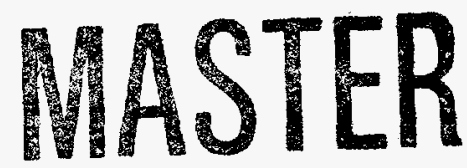




\section{DISCLAIMER}

This document was prepared as an account of work sponsored by an agency of the United States Government. Neither the United States Government nor the University of California nor any of their employees, makes any warranty, express or implied, or assumes any legal liability or responsibility for the accuracy, completeness, or usefulness of any information, apparatus, product, or process disclosed, or represents that its use would not infringe privately owned rights. Reference herein to any specific commercial products, process, or service by trade name, trademark, manufacturer, or otherwise, does not necessarily constitute or imply its endorsement, recommendation, or favoring by the United States Government or the University of California. The views and opinions of authors expressed herein do not necessarily state or reflect those of the United States Government or the University of California, and shall not be used for advertising or product endorsement purposes. 


\section{DISCLAIMER}

Portions of this document may be illegible in electronic image products. Images are produced from the best available original document. 


\title{
Absorption of Laser Light in Overdense Plasmas by Sheath Inverse Bremsstrahlung
}

\author{
T.-Y. Brian Yang, William L. Kruer, and Richard M. More \\ Lawrence Livermore National Laboratory
}

The original sheath inverse bremsstrahlung model [P. J. Catto and R. M. More, 1977] is modified by including the $\mathbf{v} \times \mathrm{B}$ term in the equation of motion. It is shown that the present results are significantly different from those derived without the $\mathrm{v} \times \mathrm{B}$ term. The $\mathrm{v} \times \mathrm{B}$ term is also important in interpreting the absorption mechanism. If the $v \times \mathbf{B}$ term were neglected, the absorption of the light would be incorrectly interpreted as an increase in the transverse electron temperature. This would violate the conservation of the transverse components of the canonical momentum, in the case of a normally incident laser light. It is also shown that both the sheath inverse bremsstrahlung and the anomalous skin effect are limiting cases of the same collisionless absorption mechanism. Finally, results from PIC plasma simulations are compared with the absorption coefficient calculated from the linear theory. 


\section{Introduction}

In recent years, there have been increasing interests in the interaction of short laser pulses with overdense plasmas ${ }^{1-12}$. For sufficiently short laser pulses, the hydrodynamics motion of the heated target does not play a dominant role, and the production of high-density plasmas with sharp density gradient become feasible. One topic of great interest is to study the dependence of the light absorption on the laser intensity and the plasma temperature in such plasmas. It has been observed in experiments ${ }^{1-3}$ that, starting from sufficiently low intensity, the absorption rate increases as a function of laser intensity, until it reaches the "resistivity saturation", a condition in which the electron mean free path reaches a minimum value. Further increase of the laser intensity and the plasma temperature will then cause an increase in the electron mean free path and a decrease in the absorption rate. When the electron mean free path is longer than the skin depth, theoretical studies ${ }^{13,14}$ suggest that. collisionless absorption mechanisms such as sheath inverse bremsstrahlung ${ }^{13}$ and the anomalous skin effect ${ }^{14,15}$ become important.

In this paper, we modify the original sheath inverse bremsstrahlung model ${ }^{13}$ by including the $\mathrm{v} \times \mathrm{B}$ term in the equation of motion. It will be shown that the present results are significantly different from those derived without the $\mathbf{v} \times \mathbf{B}$ term, except when the distribution function is isotropic $\left[f_{0}(\mathbf{v})=f_{0}(|\mathbf{v}|)\right]$. For an isotropic distribution function, identical results will be obtained whether or not the $\mathrm{v} \times \mathbf{B}$ term in the equation of motion is included in the derivation. However, if the $v \times \mathbf{B}$ term were neglected, the absorption of the light would be incorrectly interpreted as an increase in the transverse electron temperature. while the conservation of the transverse components of the canonical momentum requires that, after leaving the interaction region $(|x| \lesssim \delta)$, an electron should have the same transverse velocity as before it entered the interaction region. 
The present analysis also determines self-consistently the profile of the electromagnetic fields in the overdense plasma in the regime of validity for the sheath inverse bremsstrahlung model, i.e., $\omega^{2} c^{2} \gg \omega_{p}^{2} v_{e}^{2}$. By deriving the absorption coefficients for both the sheath inverse bremsstrahlung and the anomalous skin effect from the same set of equations [Eqs. (20) and (21)], it is shown, in Sec. III, that both phenomena are limiting cases of the same collisionless absorption mechanism. The sheath inverse bremsstrahlung corresponds to the limit where $\omega^{2} c^{2} \gg \omega_{p}^{2} v_{e}^{2}$, while the anomalous skin effect corresponds to $\omega^{2} c^{2} \ll \omega_{p}^{2} v_{e}^{2}$.

Finally, numerical simulations of the light absorption in overdense plasmas have been carried out, using the PIC plasma simulation code ZOHAR ${ }^{16}$. The absorption coefficients observed in the numerical simulations agree qualitatively with the values calculated from the linear theory, as illustrated by the results shown in Sec. IV.

\section{Theoretical Model and Derivation of the Sheath Inverse Bremsstrahlung Absorption}

The model consists of an overdense plasma filling the half-space $x>0$, and electromagnetic fields of the following form

$$
\begin{aligned}
& E_{y}(x, t)=\operatorname{Re}\left\{E_{0} \exp [i(k x-\omega t)]\right\}, \\
& B_{z}(x, t)=\operatorname{Re}\left\{\frac{c k}{\omega} E_{0} \exp [i(k x-\omega t)]\right\},
\end{aligned}
$$

where $E_{0}$ and $\omega$ are real-valued constants, while $k$ is a complex number with a positive imaginary part. Immobile ions, with zero density for $x<0$ and a constant density for $x>0$, are assumed to form the neutralizing background. The plasma is assumed to be highly overdense $\left(\omega_{p}^{2} \gg \omega^{2}\right)$ and the fiducial thermal velocity $v_{e}$, which characterizes the electron distribution, is sufficiently small $\left(\omega^{2} c^{2} \gg \omega_{p}^{2} v_{e}^{2}\right)$. Except in the sheath regime near $x=0$, the electron density is equal to $n_{0}$ for $x>0$ and is zero for $x<0$. When an electron hits the sheath $(x=0)$ from the right $(x>0)$, instantaneous 
specular reflection is assumed, that is the $y$ and $z$ components of the momentum remain unchanged, while the $\mathrm{x}$-component reverses with the amplitude unchanged. Since the typical time scale to reverse electron momentum in the sheath region is $1 / \omega_{p}$, which is much shorter than both the wave period $(2 \pi / \omega)$ and the transit time in the skin depth $\left(c / v_{e} \omega_{p}\right)$ in an overdense non-relativistic plasma, instantaneous reflection is a good assumption. Here, $\omega_{p}=\left(4 \pi n_{0} e^{2} / m_{e}\right)^{1 / 2}$ is the electron plasma frequency. The assumption of specular reflection requires that the sheath be one-dimensional, i.e., the length scale of the transverse variation be much longer than the width of the sheath (approximately equal to the Debye length). The present analysis also assumes that the quiver velocity $v_{\mathrm{os}}=e E_{0} / m_{e} \omega$ is much smaller than the fiducial thermal velocity $v_{e}$, so that the perturbation analysis is applicable.

For an electron located at $x^{\prime}\left(t^{\prime}\right)=x>0$, at the time $t^{\prime}=t$, with velocity $\mathrm{v}^{\prime}\left(t^{\prime}=t\right)=\mathrm{v}=v_{x} \hat{e}_{x}+v_{y} \hat{e}_{y}+v_{z} \hat{e}_{z}$, the unperturbed orbits at any earlier time $t^{\prime}<t$, in the absence of the electromagnetic fields $\left(E_{0}=0\right)$, are

$$
\begin{aligned}
& x^{(0)}\left(t^{\prime}\right)=x+v_{x}\left(t^{\prime}-t\right), \\
& \mathrm{v}^{(0)}\left(t^{\prime}\right)=\mathrm{v},
\end{aligned}
$$

for $v_{x}<0$, and

$$
\begin{aligned}
& \begin{cases}x^{(0)}\left(t^{\prime}\right)=x+v_{x}\left(t^{\prime}-t\right), & t^{\prime}>t_{r}, \\
x^{(0)}\left(t^{\prime}\right)=-x-v_{x}\left(t^{\prime}-t\right), & t^{\prime}<t_{r},\end{cases} \\
& \begin{cases}v_{x}^{(0)}\left(t^{\prime}\right)=v_{x}, & t^{\prime}>t_{r}, \\
v_{x}^{(0)}\left(t^{\prime}\right)=-v_{x}, & t^{\prime}<t_{r},\end{cases} \\
& v_{y}^{(0)}\left(t^{\prime}\right)=v_{y}, v_{z}^{(0)}\left(t^{\prime}\right)=v_{z},
\end{aligned}
$$

for $v_{x}>0$, where $t_{r}=t-x / v_{x}$ is the time of reflection.

To calculate the electron orbits correctly to the first order in $E_{0}$, the orbits are written in the following form

$$
x^{\prime}\left(t^{\prime}\right)=x^{(0)}+\operatorname{Re} \delta x\left(t^{\prime}\right), v_{x}^{\prime}\left(t^{\prime}\right)=v_{x}^{(0)}+\operatorname{Re} \delta v_{x}\left(t^{\prime}\right), v_{y}^{\prime}\left(t^{\prime}\right)=v_{y}^{(0)}+\operatorname{Re} \delta v_{y}\left(t^{\prime}\right),
$$


where the perturbed orbits $\delta x, \delta v_{x}$, and $\delta v_{y}$ satisfy the following linearized equations

$$
\begin{aligned}
\frac{d \delta v_{y}}{d t^{\prime}} & =-\frac{e}{m_{e}}\left(1-\frac{k v_{x}}{\omega}\right) E_{0} \exp \left\{i\left[k x^{(0)}\left(t^{\prime}\right)-\omega t^{\prime}\right]\right\} \\
\frac{d \delta v_{x}}{d t^{\prime}} & =-\frac{e v_{y}}{m_{e}} \frac{k}{\omega} E_{0} \exp \left\{i\left[k x^{(0)}\left(t^{\prime}\right)-\omega t^{\prime}\right]\right\} \\
\frac{d \delta x}{d t^{\prime}} & =\delta v_{x}\left(t^{\prime}\right) .
\end{aligned}
$$

For an electron moving to the left with $v_{x}<0$, Eqs. (2) and (5) readily give the perturbed orbits at the time $t^{\prime}=t$

$$
\begin{aligned}
\delta v_{y}(t) & =\frac{e E_{0}}{i m_{e} \omega} \exp [i(k x-\omega t)] \\
\delta v_{x}(t) & =\frac{e E_{0}}{i m_{e} \omega} \frac{k v_{y}}{\omega-k v_{x}} \exp [i(k x-\omega t)]
\end{aligned}
$$

For an electron moving to the right with $v_{x}>0$, the condition of specular reflection requires that $\delta v_{y}\left(t_{r}+\epsilon\right)=\delta v_{y}\left(t_{r}-\epsilon\right)$ and $\delta v_{x}\left(t_{r}+\epsilon\right)=-\delta v_{x}\left(t_{r}-\epsilon\right)$ for a small positive $\epsilon$. Making use of Eq. (3) and the specular reflection conditions, Eq. (5) is solved to give the perturbed orbits for the right-moving particle $\left(v_{x}>0\right)$ at the time $t^{\prime}=t$

$$
\begin{aligned}
& \delta v_{y}(t)=\frac{e E_{0}}{i m_{e} \omega} \exp [i(k x-\omega t)] \\
& \delta v_{x}(t)=\frac{e E_{0}}{i m_{e} \omega} \frac{k v_{y}}{\omega-k v_{x}} \exp [i(k x-\omega t)]+\frac{2 e E_{0}}{m} \frac{i k v_{y}}{\omega^{2}-k^{2} v_{x}^{2}} \exp \left[i \omega\left(\frac{x}{v_{x}}-t\right)\right] .
\end{aligned}
$$

In the absence of the electromagnetic fields $\left(E_{0}=0\right)$, the distribution function of the electron is independent of time and can be expressed as

$$
F(x, \mathrm{v}, t)= \begin{cases}n_{0} f_{0}(\mathrm{v}), & x>0 \\ 0, & x<0\end{cases}
$$

where $f_{0}$ satisfies the normalization condition $\int d^{3} v f_{0}(\mathrm{v})=1$ and the symmetry $f_{0}\left(-v_{x}, v_{y}, v_{z}\right)=f_{0}\left(v_{x}, v_{y}, v_{z}\right)$. In the presence of the electromagnetic fields, the induced current density can be calculated from the perturbed orbits in Eqs. (6) and 
(7), using the relation

$$
\begin{aligned}
j_{y} & =-n_{0} e \int d^{3} v \delta v_{y} f_{0}(\mathbf{v})+n_{0} e \int d^{3} v v_{y} \frac{\partial \delta x_{i}}{\partial x_{i}} f_{0}(\mathbf{v}) \\
& =-n_{0} e \int d^{3} v \delta v_{y} f_{0}(\mathbf{v})-n_{0} e \int d^{3} v v_{y} \frac{\partial \delta v_{i}}{\partial v_{i}} f_{0}(\mathbf{v})
\end{aligned}
$$

for $x>0$. Here, $x_{i}$ and $v_{i}(\mathrm{i}=1,2$, or 3$)$ are the $x, y$, or $z$ components of position ( $\mathrm{x}$ ) and velocity $(v)$ vectors, and a repeated index implies a summation over the index. In deriving Eq. (9), use has been made of the relation $\frac{\partial \delta x_{i}}{\partial x_{i}}+\frac{\partial \delta v_{i}}{\partial v_{i}}=0$, which can be derived from the Liouville's theorem. It is worth mentioning that the second term in the right-hand side of Eq. (9) is due to current bunching, which is characteristic of collective interactions between electromagnetic fields and plasmas (e.g., Weibel instability ${ }^{17,18}$ and cyclotron maser instability ${ }^{19}$ ). Substituting Eqs. (6) and (7) into Eq. (9) readily gives the induced current density

$$
j_{y}=\frac{i n_{0} e^{2} E_{0}}{m_{e} \omega}\left[1+\int d^{3} v \frac{k^{2} v_{y}^{2}}{\left(w-k v_{x}\right)^{2}} f_{0}(\mathrm{v})\right] \exp [i(k x-\omega t)]+j_{R},
$$

where $j_{R}$ is defined by

$$
j_{R}=-\frac{2 i n_{0} e^{2} E_{0}}{m_{e}} \int d^{2} v_{\perp} \int_{0}^{\infty} d v_{x} f_{0} \frac{k v_{y}^{2}}{\omega^{2}-k^{2} v_{x}^{2}}\left[\frac{2 k^{2} v_{x}}{\omega^{2}-k^{2} v_{x}^{2}}-i \frac{\omega x}{v_{x}^{2}}\right] \exp \left[i \omega\left(\frac{x}{v_{x}}-t\right)\right] .
$$

In the regime where $\omega^{2} c^{2} \gg \omega_{p}^{2} v_{e}^{2}$ and $\omega_{p}^{2} \gg \omega^{2}$, the $j_{R}$ term in Eq. (10) is small in comparison with the other term. Neglecting the $j_{R}$ term, the induced current $j_{y}$ can be substituted into the Maxwell's equations to determine the relation between $k$ and $\omega$, i.e.,

$$
\omega^{2}-c^{2} k^{2}=\omega_{p}^{2}\left[1+\int d^{3} v \frac{k^{2} v_{y}^{2}}{\left(w-k v_{x}\right)^{2}} f_{0}(\mathrm{v})\right]
$$

or equivalently

$$
\omega^{2}+\frac{c^{2}}{\delta^{2}}=\omega_{p}^{2}\left[1-\int d^{3} v \frac{v_{y}^{2}\left(\omega^{2} \delta^{2}-v_{x}^{2}\right)}{\left(\omega^{2} \delta^{2}+v_{x}^{2}\right)^{2}} f_{0}(\mathbf{v})\right],
$$

with $\delta=i / k$. In this regime $\left[\omega^{2} c^{2} \gg \omega_{p}^{2} v_{e}^{2}\right.$ and $\left.\omega_{p}^{2} \gg \omega^{2}\right]$, the skin depth $\delta$ is

$$
\delta=\delta_{0}+\frac{\omega_{p}^{2} \delta_{0}^{3}}{2 c^{2}} \int d^{3} v \frac{v_{y}^{2}\left(\omega^{2} \delta_{0}^{2}-v_{x}^{2}\right)}{\left(\omega^{2} \delta_{0}^{2}+v_{x}^{2}\right)^{2}} f_{0}(\mathrm{v})
$$


where $\delta_{0}=c /\left(\omega_{p}^{2}-\omega^{2}\right)^{1 / 2}$. Making use of Eq. (11), the power transfering from the laser to the plasma, per unit area of laser-plasma interface, is readily obtained

$$
\begin{aligned}
P_{\mathrm{ab}} & =\frac{1}{2} \int_{0}^{\infty} d x \operatorname{Re}\left\{\left[E_{0} \exp \left(-\frac{x}{\delta}-i \omega t\right)\right]^{*} j_{R}\right\} \\
& =\frac{2 n_{0} e^{2} E_{0}^{2} \delta^{2}}{m} \int d^{2} v_{\perp} \int_{0}^{\infty} d v_{x} \frac{v_{x} v_{y}^{2}\left(\omega^{2} \delta^{2}-v_{x}^{2}\right)}{\left(\omega^{2} \delta^{2}+v_{x}^{2}\right)^{3}} f_{0}(\mathrm{v})
\end{aligned}
$$

It is worth mentioning that a similar derivation without the $\mathbf{v} \times \mathbf{B}$ term in the equation of motion will give

$$
P_{\mathrm{ab}}=\frac{n_{0} e^{2} E_{0}^{2} \delta^{2}}{m} \int d^{2} v_{\perp} \int_{0}^{\infty} d v_{x} \frac{v_{x}^{3}}{\left(\omega^{2} \delta^{2}+v_{x}^{2}\right)^{2}} f_{0}(\mathrm{v})
$$

For an isotropic distribution function $\left[f_{0}(v)=f_{0}(|v|)\right]$, it can be shown, by carrying out the integration over the solid angle $\sin \theta d \theta d \phi$, that Eq.(16) is equivalent to Eq.(15). For general distributions function, however, Eq.(16) gives an absorption rate significantly different from Eq.(15). Moreover, if the $\mathbf{v} \times \mathbf{B}$ term had been ne-

glected, the absorption of the light would be incorrectly interpreted as an increase in the transverse ( $y$-direction) electron temperature, while the conservation of the transverse components of the canonical momentum requires that, after leaving the interaction region $(|x| \lesssim \delta)$, an electron should have the same $y$-velocity as before it entered the interaction region.

\section{Relation between the Sheath Inverse Bremsstrahlung and the Anomalous Skin Effect}

In the usual treatment of the anomalous skin effect ${ }^{14,15}$, the absorption coefficient is calculated by extending the plasma and the electromagnetic fields in the present model to the half space $x<0$ with

$$
E_{y}(-x)=E_{y}(x), B_{z}(-x)=-B_{z}(x)
$$


The discontinuity in $B_{z}$ requires a current sheet $\mathrm{J}=\hat{e}_{y} J_{0} \delta(x) \exp (-i \omega t)$, whose amplitude is determined by

$$
B_{z}\left(x=0^{+}\right)-B_{z}\left(x=0^{-}\right)=2 B_{z}\left(x=0^{+}\right)=-\frac{4 \pi J_{0}}{c} .
$$

Since an electron with $x>0$ in the extended model will have the same orbits as the corresponding electron in the original model, the two models are equivalent as far as the region $x>0$ is concerned. In the extended model, the electric field satisfies the following equation

$$
\left(c^{2} \frac{\partial^{2}}{\partial x^{2}}+\omega^{2}\right) E_{y}(x)=-4 \pi i \omega\left[j_{y}(x)+J_{0} \delta(x)\right],
$$

where $j_{y}(x)$ is the current density induced by the electromagnetic fields. Performing the Fourier transform on Eq. (19), and making use of the well-known relation ${ }^{20}$ between the induced current density $j_{y}$ and the electric neld $E_{y}^{\prime}$, it follows that

$$
\begin{aligned}
\tilde{E}_{y}(k) & =\frac{-4 \pi i \omega J_{0}}{D(\omega, k)} \\
D(\omega, k) & =\omega^{2}-c^{2} k^{2}-\omega_{p}^{2}\left[1-\int d^{3} v \frac{k v_{y}^{2}}{\omega-k v_{x}} \frac{\partial f_{0}}{\partial v_{x}}\right] .
\end{aligned}
$$

Here, $\tilde{E}_{y}(k)$ is the Fouier transform of $E_{y}(x)$, i.e.,

$$
\tilde{E}_{y}(k)=\int_{-\infty}^{\infty} d x E_{y}(x) \exp (-i k x)
$$

In the regime of the anomalous skin effect $\left(\omega^{2} c^{2} \ll \omega_{p}^{2} v_{e}^{2}\right.$ and $\left.\omega_{p}^{2} \gg \omega^{2}\right)$, and for an isotropic distribution function $\left[f_{0}(\mathbf{v})=f_{0}(|\mathbf{v}|)\right]$, the function $D(\omega, k)$ can be approximated by

$$
\begin{aligned}
D(\omega, k) & \simeq-c^{2}\left(k^{2}-\frac{i}{|k| \delta_{a s}^{3}}\right), \\
\frac{1}{\delta_{a s}^{3}} & =\frac{2 \pi^{2} \omega_{p}^{2} \omega}{c^{2}} \int_{0}^{\infty} d v v f_{0}(v) .
\end{aligned}
$$

Making use of Eq. (18), (20) and (23), the electric field at $x=0$ is readily obtained

$$
E_{y}(x=0)=\frac{4 i \omega J_{0}}{c^{2}} \int_{0}^{\infty} d k \frac{k \delta_{a s}^{3}}{k^{3} \delta_{a s}^{3}-i}=\frac{2 B_{z}\left(x=0^{+}\right) \omega \delta_{a s}}{3 \sqrt{3} c}(1-i \sqrt{3}),
$$


and so is the Poynting flux at $x=0^{+}$

$$
\frac{c}{8 \pi} \operatorname{Re}\left\{E_{y}(x=0) B_{z}^{*}\left(x=0^{+}\right)\right\}=\frac{\omega \delta_{a s}\left|B_{z}\left(x=0^{+}\right)\right|^{2}}{12 \sqrt{3} \pi} .
$$

For a laser normally incident on a highly overdense plasma, the magnetic field at $x=0$ is related to the incident electric field $\left(E_{\text {in }}\right)$ by $\left|B_{z}\left(x=0^{+}\right)\right|=2 E_{\text {in }}$; therefore, the absorption coefficient $\eta_{a s}$ of the anomalous skin effect is

$$
\eta_{a s}=\frac{8 \omega \delta_{a s}}{3 \sqrt{3} c}
$$

In the regime of the sheath inverse bremsstrahlung $\left(\omega^{2} c^{2} \gg \omega_{p}^{2} v_{e}^{2}\right.$ and $\left.\omega_{p}^{2} \gg \omega^{2}\right)$, on the other hand, the following approximation is appropriate

$$
\frac{1}{D(\omega, k)} \simeq-\frac{1}{c^{2}\left(k^{2}+\frac{1}{\delta^{2}}\right)}-\frac{\Delta}{c^{2}\left(k^{2}+\frac{1}{\delta^{2}}\right)^{2}}-\frac{\omega_{p}^{2}}{c^{4}\left(k^{2}+\frac{1}{\delta^{2}}\right)^{2}} \int d^{3} v \frac{k v_{y}^{2}}{\omega-k v_{x}} \frac{\partial f_{0}}{\partial v_{x}}
$$

where $\delta$ is defined by

$$
\frac{c^{2}}{\delta^{2}}=\omega_{p}^{2}-\omega^{2}+\Delta c^{2},
$$

and $\Delta$ is a constant to be determined by the condition that the secular term in the final result should vanish. Substituting Eq. (27) into Eq. (20) and performing the inverse Fourier transform give

$$
\begin{aligned}
E_{y}(x>0)= & \frac{1}{2 \pi} \int_{\Gamma} d k \tilde{E}_{y}(k) \exp (i k x)=\frac{2 i \omega J_{0}}{c^{2}} \int_{\Gamma} d k \frac{\exp (i k x)}{k^{2}+\frac{1}{\delta^{2}}}+\frac{2 i \omega J_{0} \Delta}{c^{2}} \int_{\Gamma} d k \frac{\exp (i k x)}{\left(k^{2}+\frac{1}{\delta^{2}}\right)^{2}} \\
& +\frac{2 i \omega \omega_{p}^{2} J_{0}}{c^{4}} \int_{\Gamma} d k \frac{\exp (i k x)}{\left(k^{2}+\frac{1}{\delta^{2}}\right)^{2}} \int^{3} v \frac{k v_{y}^{2}}{\omega-k v_{x}} \frac{\partial f_{0}}{\partial v_{x}} \\
= & 2 \pi i \frac{\omega \delta J_{0}}{c^{2}} \exp \left(-\frac{x}{\delta}\right)+\pi i \frac{\omega \delta^{2} J_{0} \Delta}{c^{2}} x \exp \left(-\frac{x}{\delta}\right)+\pi i \frac{\omega \delta^{3} J_{0} \Delta}{c^{2}} \exp \left(-\frac{x}{\delta}\right) \\
& +E_{A}(x>0),
\end{aligned}
$$

where the contour $\Gamma$ of the $d k$ integration goes from $k=-\infty$ to $k=+\infty$ along the real $k$-axis, with a small positive (negative) imaginary part when $\operatorname{Re} k<0$ ( $\operatorname{Re}$ $k>0)$, and $E_{A}(x>0)$ is defined by

$$
E_{A}(x>0)=\frac{2 i \omega \omega_{p}^{2} J_{0}}{c^{4}} \int_{\Gamma} d k \frac{\exp (i k x)}{\left(k^{2}+\frac{1}{\delta^{2}}\right)^{2}} \int d^{3} v \frac{k v_{y}^{2}}{\omega-k v_{x}} \frac{\partial f_{0}}{\partial v_{x}} .
$$


Reversing the order of the $d k$ and $d^{3} v$ integrations in Eq. (30), followed by closing the contour $\Gamma$ in the upper complex- $k$ plane $(\operatorname{Im} k>0)$, it can be shown that

$$
\begin{aligned}
E_{A}(x>0)= & 4 \pi \frac{\omega^{2} \omega_{p}^{2} J_{0}}{c^{4}} \int d^{2} v_{\perp} v_{y}^{2} \int_{0}^{\infty} d v_{x} \frac{\delta^{4} v_{x}^{2}}{\left(\omega^{2} \delta^{2}+v_{x}^{2}\right)^{2}} \frac{\partial f_{0}}{\partial v_{x}} \exp \left(i \frac{\omega x}{v_{x}}\right) \\
& -\frac{\pi \omega \omega_{p}^{2} J_{0}}{c^{4}} \int d^{3} v \frac{\delta^{2} v_{y}^{2}}{\omega \delta-i v_{x}} \frac{\partial f_{0}}{\partial v_{x}} x \exp \left(-\frac{x}{\delta}\right) \\
& -\frac{\pi \omega \omega_{p}^{2} J_{0}}{c^{4}} \int d^{3} v \frac{\delta^{3} v_{y}^{2}}{\omega \delta-i v_{x}} \frac{\partial f_{0}}{\partial v_{x}} \exp \left(-\frac{x}{\delta}\right) \\
& +\frac{\pi \omega^{2} \omega_{p}^{2} J_{0}}{c^{4}} \int d^{3} v \frac{\delta^{4} v_{y}^{2}}{\left(\omega \delta-i v_{x}\right)^{2}} \frac{\partial f_{0}}{\partial v_{x}} \exp \left(-\frac{x}{\delta}\right) .
\end{aligned}
$$

It can be readily seen from Eqs (29) and (31) that, for the secular term $[x \exp (-x / \delta)$ term] to vanish. it requires that

$$
\begin{aligned}
\Delta c^{2}=\omega^{2}-\omega_{p}^{2}+\frac{c^{2}}{\delta^{2}} & =-i \omega_{p}^{2} \int d^{3} v \frac{v_{y}^{2}}{\omega \delta-i v_{x}} \frac{\partial f_{0}}{\partial v_{x}} \\
& =\omega_{p}^{2} \int d^{3} v \frac{v_{y}^{2} v_{x}}{\omega^{2} \delta^{2}+v_{x}^{2}} \frac{\partial f_{0}}{\partial v_{x}} .
\end{aligned}
$$

It can shown, using integration by parts, that Eq. (32) is identical to Eq. (13).

Making use of Eqs. (18), (29), (31) and (32), we obtain the electric field

$$
\begin{aligned}
E_{y}(x>0)= & 2 \pi i \frac{\omega \delta J_{0}}{c^{2}} \exp \left(-\frac{x}{\delta}\right)+2 \pi i \frac{\omega^{3} \omega_{p}^{2} \delta^{5} J_{0}}{c^{4}} \int d^{3} v \frac{v_{y}^{2} v_{x}}{\left(\omega^{2} \delta^{2}+v_{x}^{2}\right)^{2}} \frac{\partial f_{0}}{\partial v_{x}} \exp \left(-\frac{x}{\delta}\right) \\
& +4 \pi \frac{\omega^{2} \omega_{p}^{2} J_{0}}{c^{4}} \int d^{2} v_{\perp} v_{y}^{2} \int_{0}^{\infty} d v_{x} \frac{\delta^{4} v_{x}^{2}}{\left(\omega^{2} \delta^{2}+v_{x}^{2}\right)^{2}} \frac{\partial f_{0}}{\partial v_{x}} \exp \left(i \frac{\omega x}{v_{x}}\right)
\end{aligned}
$$

and the Poynting flux at $x=0^{+}$

$$
\frac{c}{8 \pi} \operatorname{Re}\left\{E_{y}(x=0) B_{z}^{*}\left(x=0^{+}\right)\right\}=-\frac{\omega^{2} \omega_{p}^{2} \delta^{4}\left|B_{z}\left(x=0^{+}\right)\right|^{2}}{4 \pi c^{2}} \int d^{2} v_{\perp} v_{y}^{2} \int_{0}^{\infty} d v_{x} \frac{v_{x}^{2}}{\left(\omega^{2} \delta^{2}+v_{x}^{2}\right)^{2}} \frac{\partial f_{0}}{\partial v_{x}} .
$$

Integrating by parts over $d v_{x}$, it is readily shown that Eq. (34) implies the same absorption rate as Eq. (15). Since the magnetic field at $x=0$ is related to the incident electric field $\left(E_{\text {in }}\right)$ by $\left|B_{z}\left(x=0^{+}\right)\right|^{2}=4 E_{\mathrm{in}}^{2} c^{2} f\left(\omega^{2} \delta^{2}+c^{2}\right)$, the absorption coefficient $\eta_{s i b}$ of the sheath inverse bremsstrahlung is

$$
\eta_{s i b}=-\frac{\delta \omega^{2} \omega_{p}^{2} \delta^{4}}{c\left(\omega^{2} \delta^{2}+c^{2}\right)} \int d^{2} v_{\perp} v_{y}^{2} \int_{0}^{\infty} d v_{x} \frac{v_{x}^{2}}{\left(\omega^{2} \delta^{2}+v_{x}^{2}\right)^{2}} \frac{\partial f_{0}}{\partial v_{x}}
$$




$$
=\frac{16 \omega^{2} \delta^{2}}{c}\left[\int d^{2} v_{\perp} v_{y}^{2} \int_{0}^{\infty} d v_{x} \frac{v_{x}\left(\omega^{2} \delta^{2}-v_{x}^{2}\right)}{\left(\omega^{2} \delta^{2}+v_{x}^{2}\right)^{3}} f_{0}(\mathrm{v})\right]\left[1-\int d^{3} v \frac{v_{y}^{2}\left(\omega^{2} \delta^{2}-v_{x}^{2}\right)}{\left(\omega^{2} \delta^{2}+v_{x}^{2}\right)^{2}} f_{0}(\mathrm{v})\right]^{-1} .
$$

Here, use has been made of Eq. (13) in deriving Eq. (35).

It can be seen from the derivations of Eqs. (26) and (35) that the sheath inverse bremsstrahlung and the anomalous skin effect are two limiting cases of a more general collisionless absorption mechanism described by Eqs. (20) and (21). The sheath inverse bremsstrahlung corresponds to the limit where $\omega^{2} c^{2} \gg \omega_{p}^{2} v_{e}^{2}$, while the anomalous skin effect corresponds to $\omega^{2} c^{2} \ll \omega_{p}^{2} v_{e}^{2}$. In the intermediate regime $\left(\omega^{2} c^{2} \sim \omega_{p}^{2} v_{e}^{2}\right)$, the aborption coefficient can be obtained by performing the inverse Fourier transform of Eqs. (20) and (21).

To further elucidate the result in Eq. (35), the absorption coefficient in a plasma with Maxwellian distribution function

$$
f_{0}(\mathbf{v})=\frac{1}{\left(2 \pi v_{e}^{2}\right)^{3 / 2}} \exp \left(-\frac{|v|^{2}}{2 v_{e}^{2}}\right)
$$

is obtained in a closed analytic form

$$
\eta_{s i b}=\frac{8}{\sqrt{2 \pi}} \frac{v_{e}}{c} a\left[(a+1) \exp (a) E_{1}(a)-1\right]\left[\frac{1}{2}+\frac{\sqrt{\pi a}}{2} \exp (a) \operatorname{erfc}(\sqrt{a})\right]^{-1},
$$

where

$$
a=\frac{\omega^{2} \delta^{2}}{2 v_{e}^{2}}, E_{1}(a)=\int_{a}^{\infty} \frac{\exp (-t)}{t} d t, \operatorname{erfc}(z)=\frac{2}{\sqrt{\pi}} \int_{z}^{\infty} \exp \left(-t^{2}\right) d t .
$$

As can be seen from Eq. (37), the quantity $\eta_{s i b} c / v_{e}$ depends on the system parameters only through the dimensionless parameter $\omega \delta / v_{e}$, which is approximately the ratio of the transit time in the collisionless skin depth over the period of the incident light. This result is to be compared with the more accurate one calculated from Eqs. (20) and (21).

Substituting the maxwellian distribution defined in Eq. (36) into Eq. (21) gives

$$
D(\omega, k)=\omega^{2}-c^{2} k^{2}+\omega_{p}^{2} \frac{\omega}{\sqrt{2}|k| v_{e}} Z\left(\frac{\omega}{\sqrt{2}|k| v_{e}}\right)
$$


where $Z(x)$ is the plasma dispersion function ${ }^{21}$. For a real-valued $x$, the function $Z(x)$ can be expressed $a^{21}$

$$
Z(x)=i \sqrt{\pi} \exp \left(-x^{2}\right)-2 \exp \left(-x^{2}\right) \int_{0}^{x} d t \exp \left(t^{2}\right)
$$

It is then readily shown by the inverse Fourier transform of Eq. (20) that

$$
E_{y}(x=0)=-\frac{i \omega c}{\pi} B_{z}\left(x=0^{+}\right) \int_{-\infty}^{\infty} d k \frac{1}{c^{2} k^{2}-\omega^{2}-\omega_{p}^{2} \frac{\omega}{\sqrt{2}|k| v_{e}} Z\left(\frac{\omega}{\sqrt{2}|k| v_{e}}\right)} .
$$

For a highly overdense plasma $\left(\omega_{p}^{2} \gg \omega^{2}\right)$, the magnetic field at $x=0$ is related to the incident electric field $\left(E_{\mathrm{in}}\right)$ by $\left|B_{z}\left(x=0^{+}\right)\right|^{2}=4 E_{\mathrm{in}}^{2}$. Changing the integration variable to $\xi=\omega / \sqrt{2}|k| v_{e}$, the absorption coefficient $\eta_{a b}$ is then readily derived from Eq. (41)

$$
\eta_{a b}=\frac{8 \sqrt{2}}{\pi} \frac{v_{e}}{c} \operatorname{Im}\left[\int_{0}^{\infty} d \xi \frac{1}{1-\frac{\xi^{3}}{b^{2}} Z(\xi)}\right],
$$

where $b^{2}=\omega^{2} c^{2} / 2 \omega_{p}^{2} v_{e}^{2}$.

Plotted in Fig. 1 are the quantities $\eta_{a b} c / v_{e}$ (solid line) calculated numerically from Eq. (42), $\eta_{s i b} c / v_{e}$ (dotted line) calculated from Eq. (37), and $\eta_{c s} c / v_{e}$ (dashed line) defined in Eq. (26). The curves are plotted versus $\omega^{2} c^{2} / \omega_{p}^{2} v_{e}^{2}$. As mentioned earlier, the sheath inverse bremsstrahlung (SIB, dotted line) corresponds to the regime where $\omega^{2} c^{2} \gg \omega_{p}^{2} v_{e}^{2}$, while the anomalous skin effect corresponds to $\omega^{2} c^{2} \ll \omega_{p}^{2} v_{e}^{2}$ (ASE, dashed line). It can be seen that both $\eta_{s i b} c / v_{e}$ and $\eta_{a s} c / v_{e}$ are in good agreement with $\eta_{a b} c / v_{e}$, in their respective regimes of validity. It is worth reiterating that, for an isotropic distribution function $\left[f_{0}(\mathbf{v})=f_{0}(|\mathbf{v}|)\right]$, such as the one in Eq. (36), an identical absorption coefficient would be obtained whether or not the $\mathrm{v} \times \mathrm{B}$ term in the equation of motion was included in the derivation. However, neglecting the $\mathbf{v} \times \mathbf{B}$ term will lead to an incorrect interpretation of the absorption mechanism. 


\section{Numerical Simulations of the Light Absorp- tion in Overdense Plasmas}

To examine the validity of the absorption coefficient derived in the previous section, several runs of numerical simulations have been carried out, using the PIC plasma simulation code $\mathrm{ZOHAR}^{16}$. In all the simulations, the electric field of the incident light $E_{\text {in }}$ satisfies $E_{\text {in }}^{2} \ll \pi n_{0} m_{e} v_{e}^{2}$ so that the qiver velocity of electrons in the overdense plasma is smaller than the thermal velocity, as was assumed in the analytical derivation of the absorption coefficient. The 1-D simulations are set up as follows: (i) The electromagnetic fields can vary only in the $x$-direction. The length of the simulation region is $l_{x}=40 c / \omega_{p}$. (ii) The boundary conditions of the electromagnetic fields at $x=0$ correspond to a normally incident circularly polarized plane wave. The amplitude of the wave gradually increases from zero at the beginning of the simulation $(t=0)$ to a value of $e E_{i n} / \omega m_{e} c$ at the end of the fifth wave period. The amplitude

of the incident wave remains constant thereafter. (iii) The wave impedances of the boundaries are chosen such that the outgoing waves will be completely reflected at $x=l_{x}$ and completely transmitted at $x=0$. (iv) The initial electron density is zero for $0<x<l_{x} / 2$, and is equal to a constant value $n_{0}$ for $l_{x} / 2<x<l_{x}$. Immobile neutralizing background charge density with the same profile is imposed to insure charge neutrality at $t=0$. (v) At $t=0$, simulation particles are loaded uniformly in the region $l_{x} / 2<x<l_{x}$, with Maxwellian distribution [Eq. (36)] in the velocity space. (vi) During the simulation, particles that hit the boundary at $x=0$ leave the system, while those that hit the boundary at $x=l_{x}$ are re-emitted according to the Maxwellian distribution with the same thermal velocity $v_{e}$ as the initial distribution function.

Three sets of simulations have been carried out with the initial thermal velocity $v_{e}$ equal to $0.1 c, 0.05 c$, and $0.025 c$, respectively. Each set consists of simulations with 
different values of $\omega / \omega_{p}$. The simulation parameters for the set with $v_{e} / c=0.1$ are: (i) 400 equally spaced grid points for the electromagnetic fields; (ii) the steady-state value of laser electric field $e E_{\text {in }} / \omega m_{e} c=0.05$; (iii) 12,800 simulation particles; (iv) the time step $\Delta t=0.08 / \omega_{p}$. Each time we reduced the value of $v_{e} / c$ by a half (from $v_{e} / c=0.1$ to $v_{e} / c=0.05$, and then to $\left.v_{e} / c=0.025\right)$, we doubled the number of simulation particles and grid points, and reduced the time step and the steady-state laser electric field $e E_{\text {in }}$ by a half.

Figure 2 shows the absorption coefficients observed in the simulations in comparison with the absorption coefficients calculated numerically from Eq. (42). Qualitative agreement between the simulations and the linear theory can be seen in Fig. 2. For the simulations with the parameter $\omega^{2} c^{2} / \omega_{p}^{2} v_{e}^{2}$ close to unity, the observed absorption coefficients are higher than the estimates from the linear theory. A possible cause of the discrepancy is that the linear theory assumes instant reflection in the sheath region, which may not be a good approximation in those simulations. This explanation is supported by the trend that the simulations with smaller $v_{e} / c$ agree better with the linear theory, as smaller $v_{e} / c$ for a given $\omega^{2} c^{2} / \omega_{p}^{2} v_{e}^{2}$ means shorter reflection time (of order $\left.1 / \omega_{p}\right)$ relative to the wave period $(2 \pi / \omega)$.

In the simulations, the flows of the electromagnetic energy and the electron kinetic energy through the boundaries at $x=0$ and $x=l_{x}$ are taken in to account; therefore, what is shown in Fig. 2 corresponds to the net transfer of the electromagnetic energy into the electron kinetic energy. For most of the simulations with $v_{e} / c=0.1$ or $v_{e} / c=0.05$, the total (electromagnetic plus kinetic) energy is conserved very well in the simulation, with the change in the total energy $\left(\Delta E_{\text {total }}\right)$ less than $10 \%$ of the net increase in the electron kinetic energy $\left(\Delta E_{e}\right)$. The exceptions are the cases with $\omega^{2} c^{2} / \omega_{p}^{2} v_{e}^{2}=20.0$ and $\omega^{2} c^{2} / \omega_{p}^{2} v_{e}^{2}=0.05$, for which $\Delta E_{\text {total }} / \Delta E_{e} \sim 20 \%$. For the simulations with $v_{e} / c=0.025, \Delta E_{\text {total }} / \Delta E_{e}$ is also about $20 \%$. 


\section{Summary and Discussion}

The sheath inverse bremsstrahlung model ${ }^{13}$ was modified by including the $\mathbf{v} \times \mathbf{B}$ term in the equation of motion. The results are significantly different from those derived without the $v \times B$ term, except when the distribution function is isotropic $\left[f_{0}(v)=\right.$ $\left.f_{0}(|v|)\right]$. For an isotropic distribution function, identical results would be obtained whether or not the $\mathbf{v} \times \mathbf{B}$ term in the equation of motion was included in the derivation. However, if the $\mathbf{v} \times \mathbf{B}$ term had been neglected, the absorption of the light would be incorrectly interpreted as an increase in the transverse electron temperature, while the conservation of the transverse components of the canonical momentum requires that, after leaving the interaction region $(|x| \lesssim \delta)$, an electron should have the same transverse velocity as before it entered the interaction region.

It was also shown that the sheath inverse bremsstrahlung is related to the anomalous skin effect in the sense that both are limiting cases of a more general collisionless absorption mechanism described by Eqs. (20) and (21). The sheath inverse bremsstrahlung corresponds to the limit where $\omega^{2} c^{2} \gg \omega_{p}^{2} v_{e}^{2}$, while the anomalous skin effect corresponds to $\omega^{2} c^{2} \ll \omega_{p}^{2} v_{e}^{2}$. In the intermediate regime $\left(\omega^{2} c^{2} \sim \omega_{p}^{2} v_{e}^{2}\right)$, the aborption coefficient can be obtained by performing the inverse Fourier transform of Eqs. (20) and (21).

Numerical simulations of the light absorption in overdense plasmas have been carried out, using the PIC plasma simulation code $\mathrm{ZOHAR}^{16}$. The absorption coefficients observed in the numerical simulations are in qualitative agreement with the lineartheory values. However, some simulations showed absorption coefficients significantly higher than the linear-theory results. A possible explanation of the discrepancy is that the assumption of instant reflection made in the linear theory may not apply very well in those simulations. It should be reiterated that the present analysis assumes that the electric field of the incident light $E_{i n}$ satisfies $E_{i n}^{2} \ll \pi n_{0} m_{e} v_{e}^{2}$, so that the qiver 
velocity of electrons in the overdense plasma is smaller than the thermal velocity. Moreover, the present model assumes normal incidence for the laser light. Studies of the cases of oblique incidence and of intense incident laser light $\left(E_{i n}^{2} \gg \pi n_{0} m_{e} v_{e}^{2}\right)$ will be subjects of future investigations.

\section{Acknowledgments}

We are grateful for valuable discussions with S. C. Wilks, A. B. Langdon, R. S. Walling, and Z. Zinamon. The research described here was performed under the auspices of the U.S. Department of Energy by the Lawrence Livermore National Laboratory under contract No. W-7405-ENG-48. 


\section{References}

[1] H. M. Milchberg, R. R. Freeman, S. C. Davey, and R. M. More, Phys. Rev. Lett., 61, 2364 (1988).

[2] J. C. Kieffer et al., Phys. Rev. Lett., 62, 760 (1989).

[3] R. Fedosejevs et al., Appl. Phys. B, 50, 79 (1990).

[4] R. Fedosejevs et al., Phys. Rev. Lett., 64, 1253 (1990).

[5] M. M. Murnane, H. C. Kapteyn, and R. W. Falcone, Phys. Rev. Lett., 62, 155 (1989).

[6] D. Kühlke, U. Herpes, and D. von der Linde, Appl. Phys. Lett. 50, 1785 (1987).

[7] C. H. Nam et al., Phys. Rev. Lett., 59, 2427 (1987).

[8] D. G. Stearns, O. L. Landen, E. M. Campbell, and J. H. Scofield, Phys. Rev. A, 37, 1684 (1988).

[9] G. Kühnle, F. P. Schäfer, S. Szatmari, and G. D. Tsakiris, Appl. Phys. B, 47, 361 (1988).

[10] J. A. Cobble et al., Phys. Rev. A, 39, 454 (1989).

[11] H. W. K. Tom and O. R. Wood, II, Appl. Phys. Lett. 54, 517 (1989).

[12] S. E. Harris and J. D. Kmetec, Phys. Rev. Lett., 61, 62 (1988).

[13] P. J. Catto and R. M. More, Phys. Fluids, 20, 704 (1977).

[14] W. Rozmus and V. T. Tikhonchuk, Phys. Rev. A, 42, 7401 (1990). 
[15] E. M. Lifshitz and L. P. Pitaevskii, Physical Kinetics (Pergamon, Oxford, 1981).

[16] A. B. Langdon and B. F. Lasinski, Meth. Comput. Phys. 16, 327 (1976).

[17] E. S. Weibel, Phys. Rev. Lett. 2,83 (1959).

[18] F. F. Chen, Introduction to Plasma Physics and Controlled Fusion, Vol. 1 (Plenum, New York, 1983), p. 223.

[19] K. R. Chu and J. L. Hirshfield, Phys. Fluids, 21, 461 (1978).

[20] R. C. Davidson, Handbook of Plasma Physics, Vol. 1, edited by A. A. Galeev and R. N. Sudan, (North-Holland, New York, 1983), pp. 521-585.

[21] B. D. Fried and S. D. Conte, The Plasma Dispersion Function (Academic, New York, 1961). 


\section{Figure Captions}

Fig. 1 The quantities $\eta_{a b} c / v_{e}$ (solid line) calculated numerically from Eq. (42), $\eta_{s i b} c / v_{e}$ (dotted line) calculated from Eq. (37), and $\eta_{a s} c / v_{e}$ (dashed line) defined in Eq. (26), plotted versus $\omega^{2} c^{2} / \omega_{p}^{2} v_{e}^{2}$.

Fig. 2 The absorption coefficents times $c / v_{e}$ observed in the PIC simulations are plotted in comparison with the linear-theory results. 


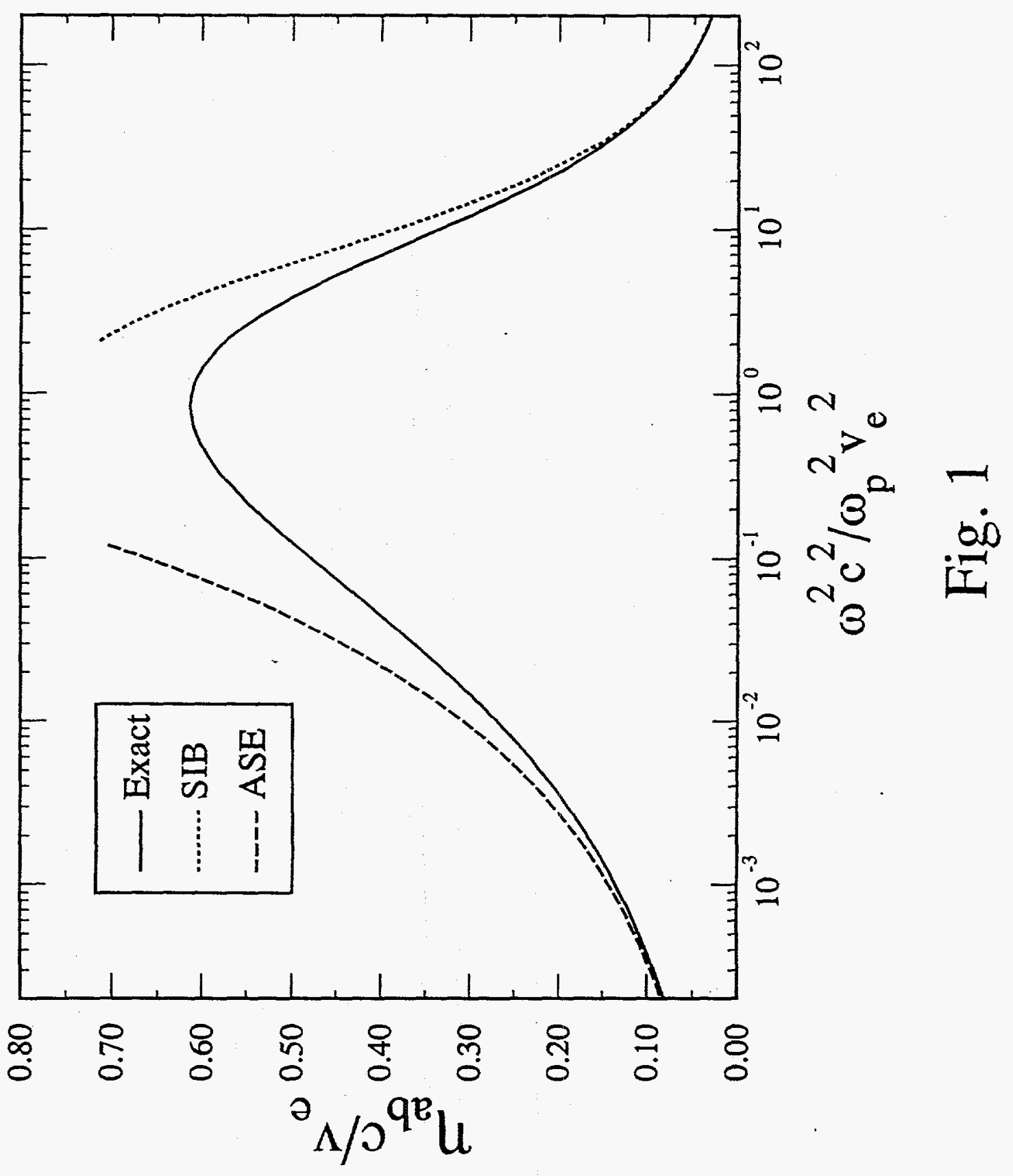




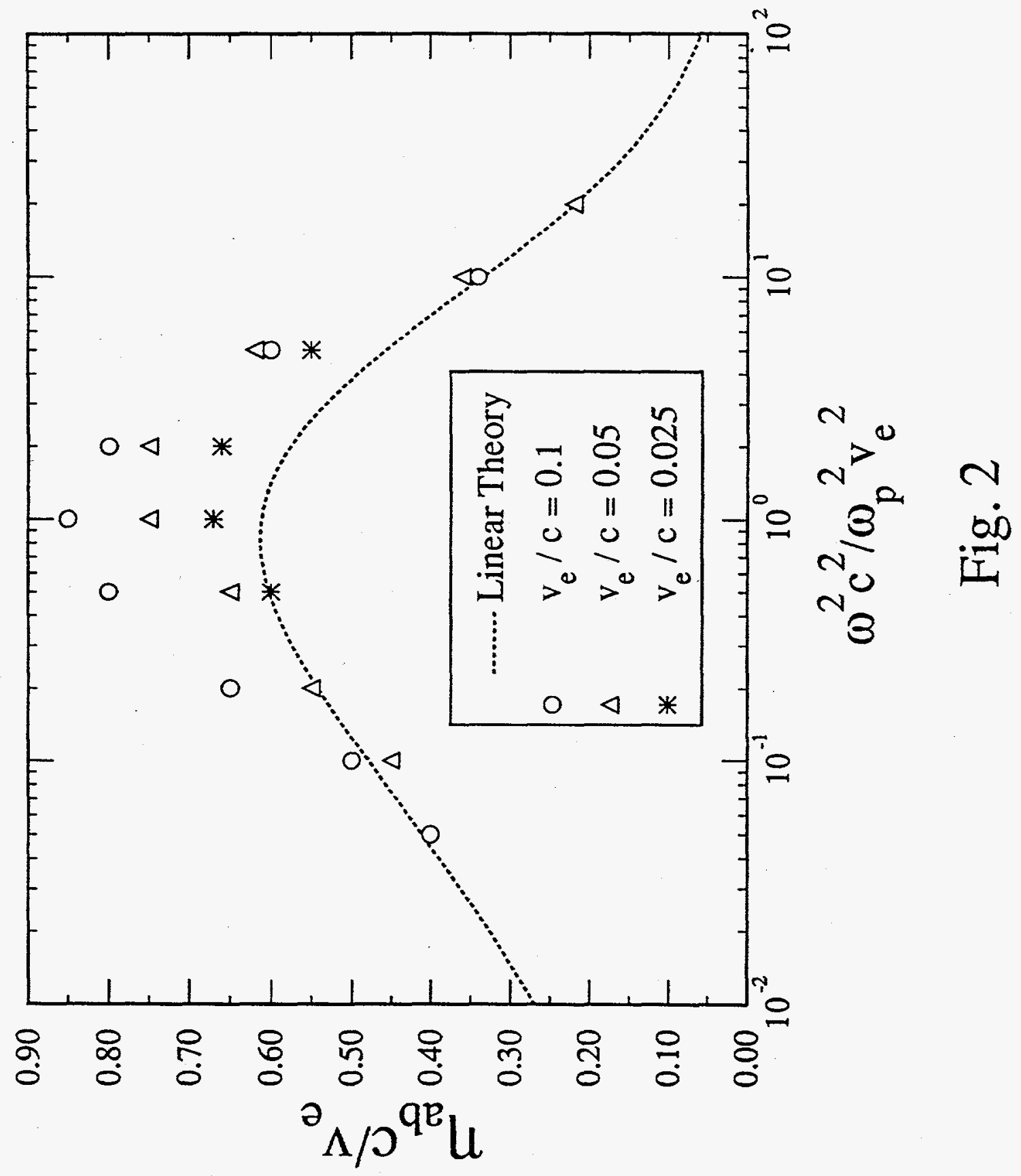

